\title{
Applying diffuse ultrasound under dynamic loading to improve closed crack characterization in concrete
}

\author{
C. Payan ${ }^{1}$, A. Quiviger ${ }^{1,2}$, V. Garnier ${ }^{1}$, J.F. Chaix ${ }^{1}$, J. Salin ${ }^{2}$ \\ ${ }^{1}$ Aix Marseille Université, Laboratory of Mechanics and Acoustics (LMA CNRS UPR 7051), 31 chemin Joseph-Aiguier \\ 13402 Marseille cedex 20, France \\ cedric.payan@univ-amu.fr
}

${ }^{2}$ EDF R\&D STEP, 6 quaiWatier, BP 49, 78401 Chatou, France

\begin{abstract}
Recent studies show the ability of diffuse ultrasound to characterize surface breaking cracks in concrete. However, derived parameters are sensitive to the presence of partially opened zones along the crack whose pattern may differ from one sample to another. The aim of this letter is to study the variation of diffuse ultrasound parameters while the sample is driven by a low frequency bending load which alternatively opens and closes the crack, allowing to access supplementary information about its morphology. The results show the sensitivity of the method for various crack depths and highlight its potential for concrete nondestructive evaluation.
\end{abstract}

PACS numbers: 43.20.Fn, 43.25.Lj

\section{Introduction}

Concrete is a highly complex material influenced by its environment during its life. In Civil Engineering structures, the multi-scale nature of concrete damage ranges from the structure scale $(\sim \mathrm{m}:$ large cracks) to the microscopic scale $(\sim \mathrm{nm}$ : chemical reactions). This paper focuses on the detection and characterization of surface breaking cracks present in the first centimeters of concrete, i.e. the concrete cover. This layer of concrete protects the rebar from environmental aggression $\left(\mathrm{CO}_{2}\right.$, water, chemical agents...) which can lead to a corrosion of the latter. Consequently, the early detection of cracks in concrete structures is challenging, especially in nuclear energy structures, for which the cost of repairs increases exponentially in time ${ }^{1}$.

Several nondestructive methods are employed for probing concrete mechanical properties, but the only one directly related with the elastic properties is ultrasound. The use of elastic waves for crack characterization is standardized ${ }^{2}$. However, its requirements, based on low frequency wave (below $100 \mathrm{kHz}$ ) time of flight measurements, can only detect a large opened crack. To decrease the detection threshold, the use of higher frequencies would be welcomed but, in this frequency range, wavelengths are on the order scale of the aggregates size which implies a strong multiple scattering phenomena making it impossible to use standard signal processing. The basic idea is to take advantage of the information carried into the coda (term used in seismology to describe the late arrivals of seismic waves on seismograms after an earthquake) of these signals to improve macro crack characterization. Recent studies highlight the potential of using coda waves for various topics related to concrete evaluation. Most of them are related with Coda Wave Interferometry, a method developed by Snieder et al. ${ }^{3}$ to measure small changes in the earth crust. Payan et al. ${ }^{4}$ evaluate the third order elastic constants of concrete and Zhang et al. ${ }^{5}$ improve the method by taking into account the influence of temperature changes during experiments. In the multiple scattering regime, the ultrasonic energy propagated in concrete follows a diffusion equation ${ }^{6}$ driven by two parameters: the diffusivity $D$, and the dissipation $\sigma$. The diffusivity (unit $\mathrm{m}^{2} \mathrm{~s}^{-1}$ ) is related to the composition. It depends on the density of the aggregates, their geometry and their average spacing. The dissipation (unit $\mathrm{s}^{-1}$ ) is an indicator of the viscoelastic properties of the medium, which in the case of concrete are mainly related to the cement paste.

The study of diffuse ultrasound in the presence of cracks in concrete is reported in the literature. Ramamoorthyet al. ${ }^{7}$, study the influence of the depth of notches (simulated cracks with no contact between its lips) on diffuse ultrasound. Jacobs et al. ${ }^{8}$ show the ability to follow self-healing of artificially opened surface breaking cracks. Quiviger et al. show both experimentally ${ }^{9}$ and numerically ${ }^{10}$ the feasibility of using diffuse ultrasound for real macro cracks characterization. 
These studies show the sensitivity of diffuse ultrasound to the presence of opened zone either for notches or opened cracks. This is also the case for closed cracks which exhibit partially opened zone along its lips ${ }^{9,10}$.

The aim of this paper is to acquire information about the closed part of the crack. Within that goal, the superimposition of a low frequency dynamical loading to diffuse ultrasound is proposed. It thus allows to probe the crack at various tension and compression states. The idea of probing the material during a dynamic loading (namely Dynamic Acouto Elastic Testing) was developed by Renaud et al. ${ }^{11}$. It was applied to the measurement of nonlinear elastic and dissipative constants of materials. The main interest of this method is its ability to probe the nonlinearity of a material at several load level. It allows some interesting nonlinear signatures to be identified ${ }^{11,12}$. The following sections present the samples and performed experiments. The results are exposed and discussed regarding the nature of the crack and its dynamical behavior.

\section{Experiments}

The samples studied here are those employed by Quiviger et $\mathrm{al}^{9}$. Four samples $\left(15 \times 15 \times 60 \mathrm{~cm}^{3}\right)$ containing real closed cracks at various depth $(0,1,3$ and $5.5 \mathrm{~cm})$ are available. These real cracks were designed by the use of a three point bending load and initiated by a $1 \mathrm{~cm}$ depth and $3 \mathrm{~mm}$ width notch at the sample center [Fig.1(a-b)]. The load was released when the desired crack depth was reached. It is important to notice that the resulting cracks are completely closed and not visible by naked eye. The crack visible Fig.1(b) was revealed by liquid penetrant inspection.

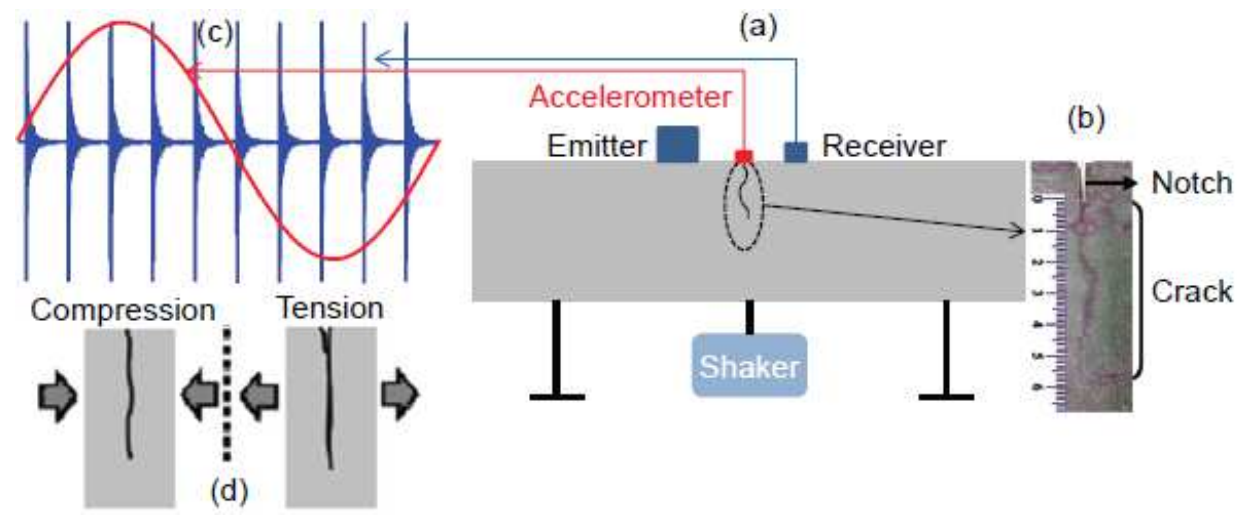

Fig.1.(Color online) (a) Experimental scheme. (b) Zoom on the $5.5 \mathrm{~cm}$ crack. (c) Schematic of recorded signals which does not match real signals. It is shown for explaining the method. (d) Behavior of the crack during dynamic bending.

Diffuse ultrasound is scrupulously measured with the protocol described by Quivigeret al. ${ }^{9}$. Ultrasound transducers are placed in contact with the upper surface of the sample, on both sides of the crack as shown in Fig. 1(a) with a constant 60 $\mathrm{mm}$ separation between them. The transmitter (Panametrics V101, diameter $25.4 \mathrm{~mm}$ ) has a central frequency of $500 \mathrm{kHz}$, and is driven by a pulsed source. The receiver is a small diameter $(4 \mathrm{~mm})$, broad-band transducer centered at $500 \mathrm{kHz}$. The only change is the superimposition of the low frequency generated by the shaker, recorded by the accelerometer [Fig.1(a,c)]. Note that the shaker is driven at constant force $(30 \mathrm{~N})$ for each sample. The sequence of pulses sent to the emitter is designed with the maximal repetition rate and making sure that the received high frequency signal does not overlap with the following. The low frequency (LF) is set so as to be uncorrelated from the pulses repetition rate $(100 \mathrm{~Hz})$ and as low as possible to be able to reasonably assume the LF amplitude to be constant over the propagating time. Additionally, the efficiency of the shaker at low frequency is taken into account, resulting in a LF frequency of $137 \mathrm{~Hz}$. This forced bending load allows the crack to be alternatively in tension and in compression while diffuse ultrasound probes the crack as shown [Fig.1(d)].

The total recorded signal length is $1 \mathrm{~s}$, with a sampling frequency of $20 \mathrm{MHz}$, averaged 500 times to increase signal to noise ratio. Each high frequency is isolated as shown in Fig.2. It is made possible because the trigger of the high frequency is also recorded but not shown for clarity. The Arrival Time of the Maximum Energy (ATME) is evaluated by fitting the energy curve [Fig.2(b)] with the 2D solution of the diffusion equation" 
$\log \left(\langle E(r, t, f\rangle)=C_{0}-\log (D t)-\frac{r^{2}}{4 D t}-\sigma t\right.$,

Where $E$ is the energy if the considered frequency band, $r$ is the distance between transducers, $C_{0}$ is a constant depending on the energy injected by the source. The energy envelope is determined by time-frequency analysis. Diffusion parameters are then evaluated by fitting the 2D solution from the received signals with a time window of $50 \mu$ s and a frequency window of $80 \mathrm{kHz}$. Each time window have a $90 \%$ overlap with the preceding window. The ATME is derived in frequency band for which a maximum quantity of energy is transmitted [240-320 kHz]. For further details about fitting procedure and signal processing, the reader is invited to refer to Quiviger et al. ${ }^{9}$. Each ATME is then related with the low frequency amplitude [noted A in Fig.2(b)] as the average amplitude during the diffusion process. The acceleration amplitude is converted to displacement $u$ in steady state conditions by $u=-\ddot{u} /\left(4 \pi^{2} f^{2}\right)$, where $f$ is the LF frequency. The only evaluated parameter is ATME due to the fact that it is known to be the more sensitive and the more robust one to probe cracks 9 .
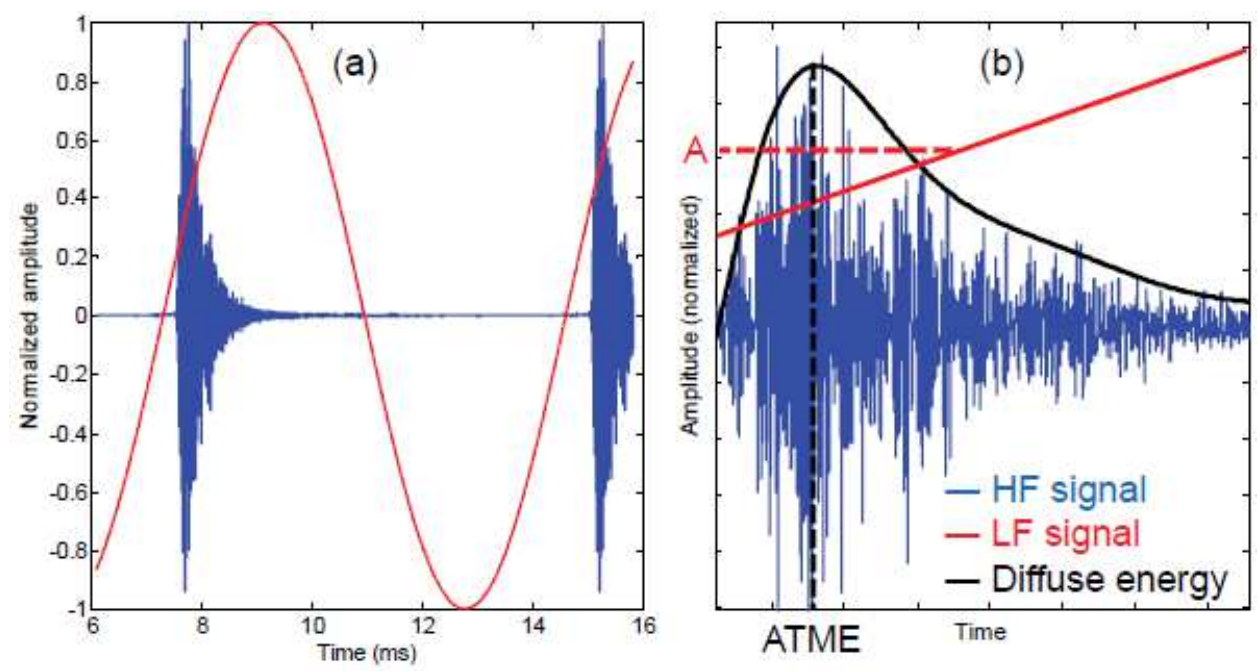

Fig.2.(Color online) (a) Experimental HF and LF signals. (b) ATME and LF amplitude evaluation.

\section{Results and discussion}

The evolution of ATME as a function of the dynamic displacement is shown Fig.3. Several observations arise from that curves. Hysteresis is present in each case, even in the sample with no crack [Fig.3(a)]. As a reminder, this sample, like other ones, has a $1 \mathrm{~cm}$ notch. One can notice that the $3 \mathrm{~cm}$ cracked [Fig.3(c)] sample does not exhibit as much hysteresis as the other ones and that the $5.5 \mathrm{~cm}$ cracked sample exhibits a particular behavior. The slope increases with crack depth except for the $5.5 \mathrm{~cm}$ cracked sample for which the slope does not really relate the dynamics. The maximal excursion of ATME during a complete cycle regularly increases with crack depth.

The dynamical behavior of the crack is complex and, to our knowledge, no former study deals with this topic. ATME is shown to decrease when the contact density along the crack increases ${ }^{9,10}$. Present data are in agreement with that statement. When the crack is under tension, the contact density decreases, leading to an increase of the ATME [Fig.3(bd)]. The evolution of the ATME for the uncracked sample [Fig.3(a)] can be explained by the intrinsic nonlinearity of concrete. It is important to notice that this nonlinearity is not due to the presence of the notch which is known to be a linear type defect, i.e., detected by linear diffusion techniques ${ }^{7,9}$.

The residual uncracked depth decreases with increasing crack depth. Thus, as the shaker is driven at constant force, the maximum displacement increases with crack depth.

The presence of hysteresis may be due to the viscoelastic nature of cement as well as the hysteretic opened/closed behavior of the crack. A real crack in concrete exhibits some partial contacts all along its lips ${ }^{10}$. It is reasonable to assume that these contact zones are hysteretic during dynamical loading. However, the low hysteretic behavior of the $3 \mathrm{~cm}$ crack 
[Fig.3(c)] is probably due to a particular crack morphology which makes the open state occurring at the same stress than the closed state.
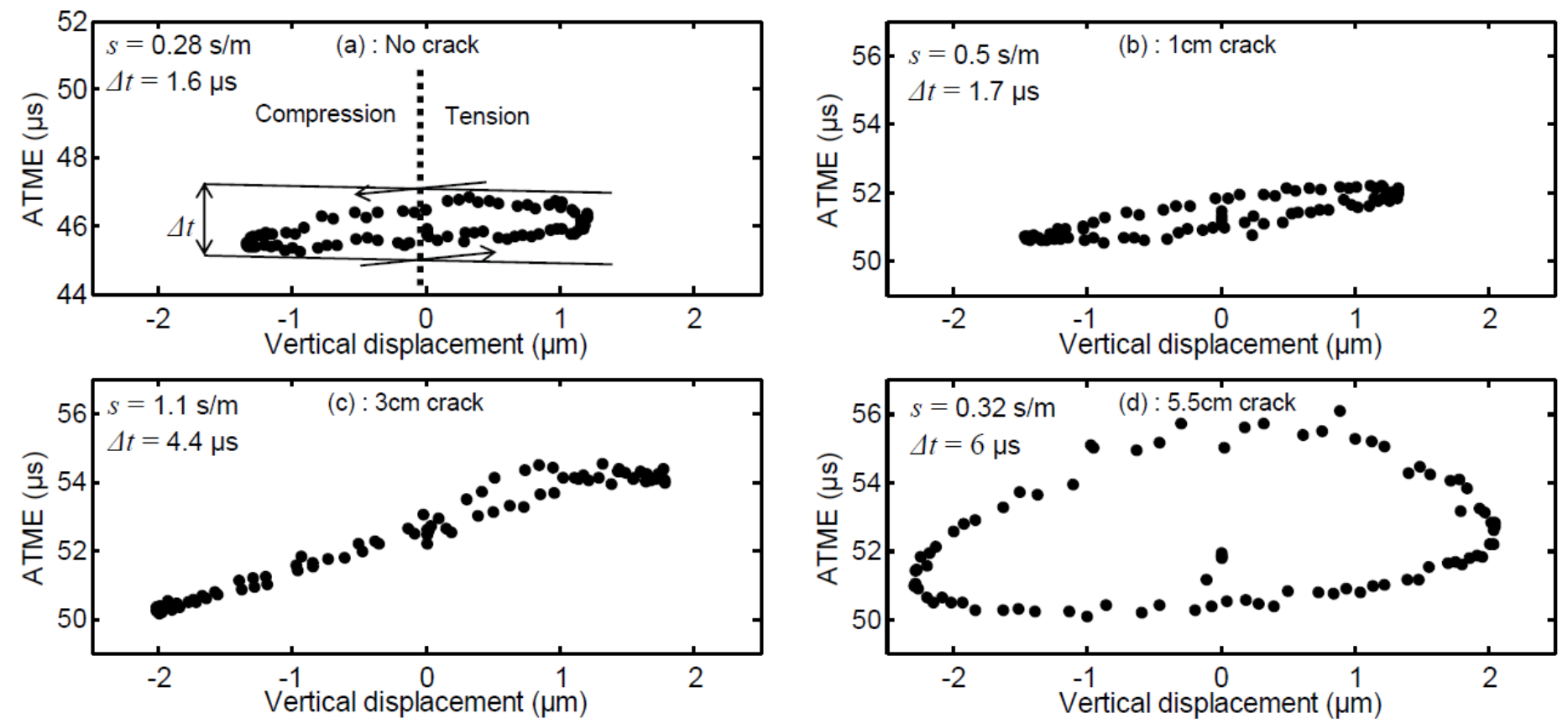

Fig.3. ATME as a function of the dynamical vertical displacement recorded by the accelerometer. Both vertical and horizontal scales are kept constant for comparison purpose.(a) Uncracked sample. The arrows indicate the displacement way which is the same for others plots. The dotted line is the linear fitting of the data with a slope $s$ and $\Delta t$ the maximal ATME excursion during a cycle. Sample with, (b) a 1cm crack, (c) $3 \mathrm{~cm}$ crack and (d) $5.5 \mathrm{~cm}$ crack.

The postulate is done that the complex signature of the $5.5 \mathrm{~cm}$ crack [Fig.3(d)] is due to its large opening during oscillations which can lead to a redistribution of contacts location. Starting from 0 to positive displacements Fig.3(d), ATME increases with a similar trend than the $3 \mathrm{~cm}$ crack [Fig.3(c)]. Due to the size of the crack compared with sample width ( half the width), when the shaker starts coming down, contact zones can be rearranged into another state while the crack remains in tension. As soon as the compression stage starts, the crack morphology comes back to its original state.

The maximum ATME excursion over a full cycle $(\Delta t)$ increases with crack depth (Fig.4). Previous studies ${ }^{7,9}$ report an ATME variation of the order of $15 \mu \mathrm{s}$ for $5 \mathrm{~cm}$ notches. Unlike notches, real closed cracks exhibit a distribution of contact along its depth, thus it is not surprising that the present $\Delta t$ remains inferior to $10 \mu \mathrm{s}$. The correlation between $\Delta t$ and crack depth may indicate that the whole crack is affected by the low frequency, even if it is not entirely opened or closed. From a qualitative point of view, one can notice the large sensitivity of $\Delta t$ to the depth of the crack with a maximum variation of about $500 \%$. Quiviger et al. ${ }^{10}$ numerically show that even a single, $1 \mathrm{~mm}$ size, contact can lead to a few microseconds ATME variation in the present samples. At the same time, the contact density makes the ATME varying in the same range ${ }^{10}$. Thus present variations and complex signatures are mostly due to a combination of these effects.

As the ATME depends on elastic diffusivity, it also depends on elastic properties of concrete ${ }^{6}$. It is worth noticing that concrete is a nonlinear medium ${ }^{13}$, meaning that its elastic behavior depends on the low frequency amplitude in experiments. The nonlinearity measured for the uncracked sample relates the "intrinsic" nonlinearity of present samples under dynamic loading. This quantity could be de-correlated from cracked samples but the physics of nonlinear phenomena under dynamic conditions in concrete is complex and not yet fully understood. For a comparison purpose, a previous study carried out on the same samples ${ }^{14}$ shown that the measured nonlinearity, using nonlinear interaction, exhibits a similar sensitivity than the modulated ATME. 


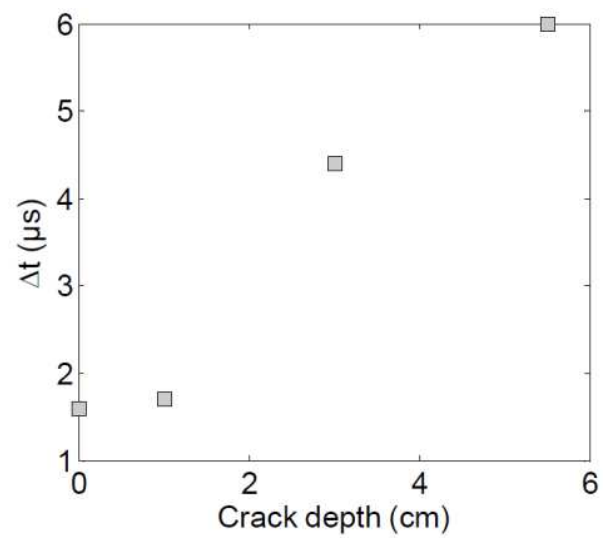

Fig.4. Evolution of the maximum ATME excursion during a cycle ( $\Delta t)$ as a function of crack depth.

\section{Conclusion}

The present letter shows the feasibility of applying diffuse ultrasound under low frequency loading to probe a real closed crack in concrete. The derived parameters exhibit a large sensitivity to crack depth $(\sim 500 \%)$. The main advantage of the method is the opportunity to get a signature of the crack depending on its morphology. As for DAE experiments, even if interesting, this kind of data are directly related to the physics but its analysis remains complex ${ }^{11,12}$. Answers should arise from the knowledge of the crack morphology and its behavior under loading. Future works will be aimed at imaging a real closed crack in concrete by $\mathrm{X}$ ray micro-tomography. In addition to these data, numerical simulation of diffuse ultrasound ${ }^{10}$ should provide the means to quantitatively relate the crack morphology to the measurements.

\section{Acknowledgements}

Authors wish to thank Electricité de France (EDF) for funding this research. The Laboratory of Materials and Durability of Constructions (LMDC) is also acknowledged for manufacturing the samples.

\section{References and links}

${ }^{1}$ P. Stephan, J. Salin, "Ageing management of concrete structure: Assessment of EDF methodology in comparison with SHM and AIEA guides," Con. Build. Mat. 37, 924-933 (2012).

${ }^{2}$ ISO 1920-7:2004, "Testing of concrete, Part 7: Non-destructive tests on hardened concrete."

${ }^{3}$ R.Snieder, A. Grêt, H. Douma, and J. Scales, "Coda wave interferometry for estimating nonlinear behavior in seismic velocity," Science 295, 2253-2255 (2002).

${ }^{4}$ C.Payan, V. Garnier, J.Moysan and P.A.Johnson, "Determination of third order elastic constants in a complex solid applying coda wave interferometry," App. Phys. Lett.94, 011904 (2009).

${ }^{5}$ Y. Zhang, O. Abraham, V.Tournat, A. Le Duff, B.Lascoup, A.Loukili, F.Grondin, O. Durand, "Validation of a thermal bias control technique for Coda Wave Interferometry (CWI),"Ultrasonics, 53(3), 658-664 (2013).

${ }^{6}$ P. Anugonda, J. Wiehn and J. Turner, “Diffusion of ultrasound in concrete,"Ultrasonics39(6), 429-435 (2001).

${ }^{7}$ S.K. Ramamoorthy, Y. Kane and J.A. Turner, "Ultrasound diffusion for crack depth determination in concrete," J. Acoust.Soc. Am. 115(2), 523-529 (2004).

${ }^{8}$ C.-W. In, R. Brett Holland, J.-Y. Kim, K.E. Kurtis, L.F. Kahn, L.J. Jacobs, "Monitoring and evaluation of self-healing in concrete using diffuse ultrasound," NDT \& E Int. 57, 36-44 (2013).

${ }^{9}$ A. Quiviger, C. Payan, J.-F.Chaix, V. Garnier, J. Salin, "Effect of the presence and size of a real macro-crack on diffuse ultrasound in concrete," NDT\&E Int. 45(1), 128-132 (2012).

${ }^{10}$ A. Quiviger, A. Girard, C. Payan, J.F. Chaix, V. Garnier, J. Salin, "Influence of the depth and morphology of real cracks on diffuse ultrasound in concrete: a simulation study," NDT \& E Int. (submitted 2013)

${ }^{11}$ G. Renaud, P.-Y. Le Bas, and P.A. Johnson, "Dynamic measures of elastic nonlinear (anelastic) behavior: Dynamic acousto-elasticity testing (DAET)," J. Geophys. Res. 117, B06202 (2012). 
${ }^{12}$ C. La Rocca, C. Payan and J. Moysan, "Evaluation of epoxy bonded joint quality using nonlinear acoustics," Proceedings of Meetings on Acoustics 10, 045001 (2010).

${ }^{13}$ C. Payan, T.J. Ulrich, P.Y. Le Bas, T.A. Saleh, M. Guimaraes, "Linear and Nonlinear Resonance Inspection Techniques and Analysis for damage characterization: Application to thermal damage in concrete," J. Acoust. Soc. Am. (submitted 2013)

${ }^{14}$ J.-P. Zardan, C. Payan, V. Garnier, J. Salin, "Effect of the presence and size of a localizednonlinear source in concrete," J. Acoust.Soc. Am. 128(1), EL38-EL42 (2010). 